\title{
The role of anti-IgE (omalizumab/Xolair) in the management of severe recalcitrant paediatric atopic eczema (ADAPT): statistical analysis plan
}

\author{
Tao Chen ${ }^{1}$, Susan Chan ${ }^{2,3}$, Gideon Lack ${ }^{3}$, Suzie $\mathrm{Cro}^{4}$ and Victoria R. Cornelius ${ }^{4^{*}}$
}

\begin{abstract}
Background: The Atopic Dermatitis Anti-lgE Paediatric Trial (ADAPT) is a trial to determine the clinical efficacy and safety of omalizumab for children with severe atopic eczema. This article describes the detailed statistical analysis plan for the ADAPT as an update to the published protocol and is submitted prior to knowing all outcomes.

Method and design: The ADAPT is a randomised, double-blind, placebo-controlled trial with a primary objective to determine whether anti-IgE reduces eczema severity as assessed by the validated eczema score (objective SCORAD) after 24 weeks of treatment in children with severe eczema. This articles outline the overall analysis principles including considerations on sample definition in each analysis, missing data, and adjusted covariates. Comparability and representativeness of the randomised groups, primary and sensitivity analyses of the primary and secondary outcomes as well as subgroup analysis are described.

Results: This prespecified statistical analysis plan has been developed to comply with international guidelines which will increase the transparency of the data analysis for the ADAPT.

Trial registration: ISRCTN, identifier: ISRCTN15090567. Registered on 3 December 2014;

EU Clinical Trials Register, EudraCT Number: 2010-020841-29. Registered on 14 May 2010. The first participant was enrolled on 15 January 2015.
\end{abstract}

Keyword: Statistical analysis plan, Eczema, Paediatric, Atopic dermatitis, Anti-lgE, Omalizumab, Randomised controlled trial, Xolair

\section{Background}

Eczema is a chronic inflammatory skin disorder with a lifetime risk of up to $22 \%$ of children by the age of $12-14$ years. The available literature suggests that anti-immunoglobulin $\mathrm{E}$ (anti-IgE) may be of benefit in the treatment of eczema from at least the age of 7 years [1-4]. Studies and case reports to date have had small numbers of participants, have not been randomised or placebo controlled, or have included a heterogenous mix of participants of different ages.

The Atopic Dermatitis Anti-IgE Paediatric Trial (ADAPT) is a trial to determine the clinical efficacy and safety of omalizumab (Xolair, Novartis) for children with severe atopic eczema. Fuller details on the rationale and the design for the trial are given in the study protocol [5]; the prespecified statistical analysis plan (SAP) has been developed and finalised without knowing the treatment allocation or treatment-related study results.

This paper describes important features of the trial design and the statistical method and procedures which need to be adhered to and performed by the statistician responsible for this study. It should be read in conjunction with the protocol.

\footnotetext{
* Correspondence: v.cornelius@imperial.ac.uk

${ }^{4}$ Imperial Clinical Trials Unit, School of Public Health, Imperial College

London, Stadium House, 68 Wood Lane, London W12 7RH, UK

Full list of author information is available at the end of the article
}

\section{Method and design}

The ADAPT is a randomised, double-blind, placebocontrolled trial of anti-IgE therapy in children with 
severe eczema who have failed topical therapy. In total, 62 children aged 4-19 years are planned to be recruited within 18 months. Participants are eligible if they have severe eczema defined as an objective SCORing Atopic Dermatitis (SCORAD) score of over 40 at assessment (detailed exclusion and inclusion criteria are in the published protocol). Participants will be individually randomised in a 1:1 ratio to two treatment arms (omalizumab and matched placebo) using minimisation to ensure the balance of total IgE $(\leq 1500$ and $>1500)$ and age $(<12$ and $\geq 12$ years). The allocation will be performed by an online randomisation system hosted at the King's Clinical Trials Unit (CTU).

\section{Primary objective}

The primary objective of this study is to assess whether omalizumab will reduce eczema severity as assessed by the validated eczema score (SCORAD) after 24 weeks of treatment in children with severe eczema.

\section{Secondary objectives}

The study will examine the influence of the study intervention on the rate of treatment failure, rate of alternative systemic therapy, quality of life, eczema severity as assessed by the Eczema Area and Severity Index (EASI), effect on co-existing allergic disease, number of eczema exacerbations, infective episodes of eczema, change in reactivity to food and aeroallergens and change in allergen-specific IgE. Detailed descriptions of the primary and secondary outcomes can be found in Table 1 as well as in the study protocol [5].

\section{Sample size}

Omalizumab is administered via subcutaneous injections which require fortnightly or monthly attendance at clinic to receive them. It is available from the manufacturers at an undisclosed cost in the United Kingdom. In order for omalizumab to be adopted into practice, a treatment effect that would make an important impact on the children's quality of life would be required. Through discussion and consultation with the funder and clinicians, a relative reduction of around $33 \%$ in symptoms was selected to be the minimum important treatment effect to detect. Given the inclusion criteria the mean baseline SCORAD score is anticipated to be 45 and we aim to detect a change in SCORAD score of 13.5 points between the treatment arms. Based upon a study by Hindley [6] we assume that the standard deviation (SD) is 15, using a significance level of $5 \%$ with $90 \%$ power, and including a $15 \%$ dropout rate we aim to recruit 62 participants (31 each to each arm).

\section{Minimum Clinically Important Difference (MCID)}

The study was powered to detect a minimum important treatment effect of a 13.5-point absolute change in objective SCORAD score taking into account the patient burden and high treatment cost. The MCID is the smallest difference in an outcome measure that represents a clinically relevant outcome to the patient, regardless of cost and burden. There is no verified MCID for SCORAD score in this severely affected paediatric population. In order to determine MCID, published studies have recommended the use of both anchor- and distribution-based methods [7]. A study by Schram et al. [8], which adopts an anchor-based approach, suggests that a MCID for the objective SCORAD score is 8.2. However, this is based on data from three RCTs on treatments for atopic eczema which included adults. The MCID reported by Schram et al. for children only, based on a subsample of $n=25$, with an average age of 9.4 years, is 9.0. Since the patients included in the study by Schram et al. also had a milder baseline severity we employed a distribution-based method using data collected from the trial to calculate a MCID. Using the data from the first 47 ADAPT patients who completed week24 assessments ( $75 \%$ of total sample size) adopting 0.7 $\mathrm{SD}$ of the change in score from baseline gives a MCID of 8.5. These MCIDs will be used to guide interpretation of the results from the primary analysis.

\section{General statistical principles}

The principle of intention-to-treat (ITT) will be the main strategy of the analysis adopted for the primary outcome and all secondary outcomes. That is, all randomised participants will be analysed in the group randomised regardless of whether the allocated study treatments were received, or whether other interventions were received and regardless of any protocol deviations or violations [9]. A safety set (SS) population will consist of participants who receive at least one dose of allocated treatment, regardless of their eligibility for the study. The harm analyses will compare the harm outcomes between the two treatment groups in the safety population.

All regression analyses will include the minimisation variables (IgE $(\leq 1500,>1500)$ and age $(<10$ or $\geq 10$ years) as covariates. This is because adjustment for these stratification factors in the randomisation process will maintain correct type I error rates [10]. Additionally, for continuous outcomes, the outcomes measured at baseline will be included in regression analysis to increase power [11].

Any examination of subgroups, not specifically identified in the SAP, will be considered exploratory in nature and will be clearly identified. All $p$ values will be twosided and the significance level is set at 5\% unless otherwise stated. 
Table 1 Outcomes and analysis models

\begin{tabular}{|c|c|c|c|}
\hline Outcome & Endpoints & Category & Analysis model \\
\hline \multicolumn{4}{|l|}{ Primary } \\
\hline Objective SCORAD & $\begin{array}{l}\text { Difference in the objective SCORAD in both } \\
\text { groups after } 24 \text { weeks of treatment }\end{array}$ & $\begin{array}{l}\text { Continuous outcome } \\
\text { measures }\end{array}$ & $\begin{array}{l}\text { Mixed-effects linear regressions } \\
\text { (primary) } \\
\text { Instrumental variable regression }\end{array}$ \\
\hline \multicolumn{4}{|l|}{ Secondary } \\
\hline Treatment failure & $\begin{array}{l}\text { Participants who have persistent severe } \\
\text { eczema despite } 2 \text { courses of rescue therapy } \\
(0.5 \text { to } 1 \mathrm{mg} / \mathrm{kg} / \text { day of orally administered } \\
\text { prednisolone for a week at a maximum dose } \\
\text { of } 40 \mathrm{mg} / \text { day, followed by a week at } 50 \% \text { of } \\
\text { this dose) }\end{array}$ & $\begin{array}{l}\text { Binary outcome } \\
\text { measures }\end{array}$ & Logistic regression model \\
\hline Alternative systemic therapy & Requirement for alternative systemic therapy & $\begin{array}{l}\text { Binary outcome } \\
\text { measures }\end{array}$ & Logistic regression model \\
\hline Eczema quality of life & $\begin{array}{l}\cdot \text { POEM } \\
\cdot(C) D L Q I\end{array}$ & $\begin{array}{l}\text { Continuous outcome } \\
\text { measures }\end{array}$ & ANCOVA \\
\hline Eczema severity & $\begin{array}{l}\text { - Subjective SCORAD } \\
\text { - EASI score }\end{array}$ & $\begin{array}{l}\text { Continuous outcome } \\
\text { measures }\end{array}$ & ANCOVA \\
\hline $\begin{array}{l}\text { Effect on co-existing allergic } \\
\text { disease }\end{array}$ & PADQLQ & $\begin{array}{l}\text { Continuous outcome } \\
\text { measures }\end{array}$ & ANCOVA \\
\hline Number of eczema exacerbations ${ }^{c}$ & $\begin{array}{l}\text { - Clinician-diagnosed exacerbation of eczema } \\
\text { or } \\
\text { - Increase on SCORAD by } 15 \text { points from last } \\
\text { recorded SCORAD with participant/parent } \\
\text { perception of worsening eczema }\end{array}$ & $\begin{array}{l}\text { Numerical outcome } \\
\text { measures }\end{array}$ & $\begin{array}{l}\text { Poisson regression } \\
\text { Negative binomial regression } \\
\text { model } \\
\text { Zero-inflated Poisson regression } \\
\text { model (as appropriate) }\end{array}$ \\
\hline Infective episodes of eczemac & $\begin{array}{l}\text { Clinician-diagnosed and -treated infective } \\
\text { episode of eczema, or clinically apparent, } \\
\text { culture-positive infective exacerbations }\end{array}$ & $\begin{array}{l}\text { Numerical outcome } \\
\text { measures }\end{array}$ & $\begin{array}{l}\text { Poisson regression } \\
\text { Negative binomial regression } \\
\text { model } \\
\text { Zero-inflated Poisson regression } \\
\text { model(as appropriate) }\end{array}$ \\
\hline Allergen-specific lg $E^{a}$ & Change in allergen-specific lgE & $\begin{array}{l}\text { Continuous outcome } \\
\text { measures }\end{array}$ & ANCOVA \\
\hline Reactivity to food and aeroallergens ${ }^{a}$ & $\begin{array}{l}\text { Change in skin-prick test reactivity to food } \\
\text { and aeroallergens }\end{array}$ & $\begin{array}{l}\text { Numerical outcome } \\
\text { measures }\end{array}$ & $\begin{array}{l}\text { Poisson regression } \\
\text { Negative binomial regression } \\
\text { model } \\
\text { Zero-inflated Poisson regression } \\
\text { model }\end{array}$ \\
\hline \multicolumn{4}{|l|}{ Safety } \\
\hline Adverse events $^{\mathrm{b}}$ & $\begin{array}{l}\text { Spontaneously reported AE will be collected } \\
\text { throughout the follow-up period }\end{array}$ & Binary outcome & Descriptive analysis \\
\hline $\begin{array}{l}\text { Urea and electrolytes, creatinine, } \\
\text { FBC, eosinophils, LFT, IgE, vitamin D, } \\
\text { iron level, bone profile }\end{array}$ & $\begin{array}{l}\text { Surveillance tests where abnormal ranges are } \\
\text { defined using the ranges specified by the } \\
\text { processing laboratory }\end{array}$ & Binary outcome & Descriptive analysis \\
\hline
\end{tabular}

${ }^{\mathrm{a}}$ Only collected at screening and 24 weeks of treatment. The remaining outcomes are collected at baseline, 4-weekly during the 24 weeks of treatment, 36 weeks and 48 weeks

${ }^{b}$ Blood test and urine samples will be collected at baseline, 24 weeks, 36 weeks and 48 weeks. Clinical observations will be examined at every visit ${ }^{c}$ Chi-square goodness-of-fit tests will be used to select the suitable model

ANCOVA analysis of covariance, IgE immunoglobulin E, SCORAD SCORing Atopic Dermatitis, PADQLQ Paediatric Allergic Disease Quality of Life Questionnaire, (C)DLQI (Children's) Dermatology Life Quality Index, EASI Eczema Area and Severity Index, AE adverse events, POEM Patient-oriented Eczema Measure, FBC full blood count, LFT liver function test

\section{Missing data}

The number and proportion of participants missing objective SCORAD values by visit number will be tabulated. The primary analysis includes all observed data and assumes the probability that missing data is not dependent on the values of the unobserved data itself, conditional on the observed values of the variables included in the analysis model (missing at random (MAR) assumption). Sensitivity analysis will explore departures from the main MAR analysis assumption for all patients on the primary outcome using a pattern-mixture multiple imputation (MI) approach [12].

Imputation under MAR will initially be performed separately within each arm following the guidance suggested by White et al. [13]. The variables in the imputation model will be the same as those in the analysis 
model without including more auxiliary variables (e.g. predictors of missingness) after taking into account the relatively small sample size of this study [14]. Imputations will then be modified to reflect departures from the MAR assumption.

We will investigate the impact of a better or poorer response than that predicted by MAR (lower/higher objective SCORAD scores) for patients with missing data. Specifically, we define $\delta$ as the postulated mean difference in the rate of change of the objective SCORAD score between the observed and unobserved cases over 24 weeks, conditional on the variables in the imputation model. For each patient we then modify the MAR imputed observations accordingly by $\delta$. Imputed data sets will be analysed using the primary analysis model. Results will be combined across imputed data sets using Rubin's rules. We will repeat the analysis for a range of $\delta$ s corresponding to $\pm 10,20,30,40$ and $50 \%$ of the rate of change of the objective SCORAD score observed over 24 weeks in all patients. We will also consider the possibility that data is missing informatively in one arm only and employ the outlined imputation approach separately by trial arm.

For baseline covariates, the amount of missing data is expected to be small. However, if this happens, in case of loss of power using observed data, mean values will be calculated from the non-missing values for the baseline variable using pooled data from both treatment groups [15]. With reference to those categorical variables, the imputed mean will be rounded up to nearest category level. This is justifiable because randomisation ensures that baseline scores are independent of treatment group and imputation keeps the statistical efficiency in the estimation of the treatment effect.

For those missing items within questionnaires, we firstly use the missing value guidance provided for questionnaires. If no guidance is provided we will then impute the missing values using the mean of the observed items within the same subscale if $20 \%$ or fewer items are missing. The scale score will be calculated based on the complete values and these replacements [16]. If more than $20 \%$ of items are missing in the questionnaire, multiple imputation will be used as discussed above.

\section{Statistical analysis \\ Trial profile}

A Consolidated Standards of Reporting Trials (CONSORT) flow chart will be constructed (see Fig. 1). This will include the number of eligible patients, the number of patients agreeing to enter the trial, the number of participants withdrawing and lost to follow-up, the number

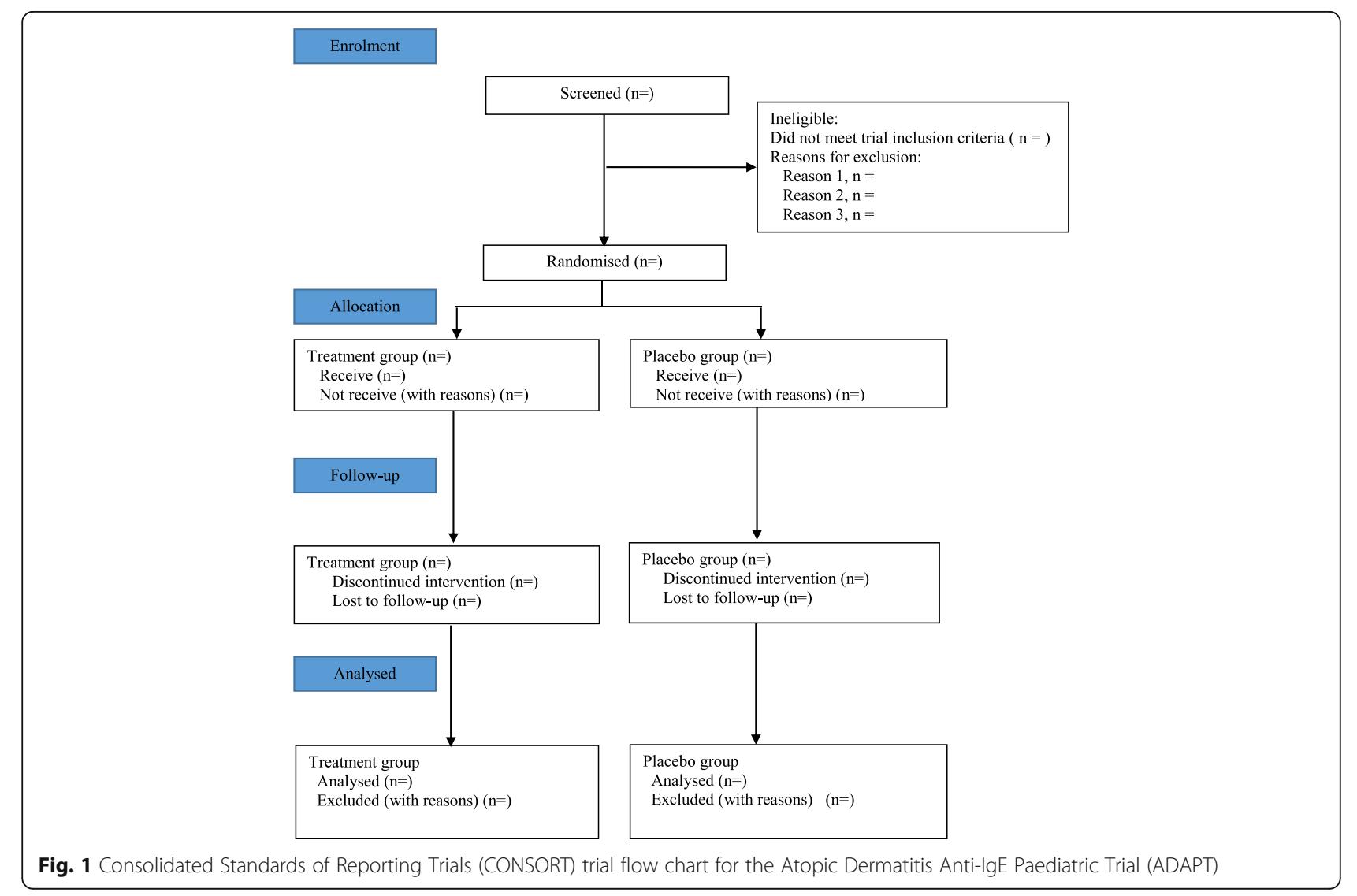


continuing through the trial, and the number included in the analyses.

\section{Comparability/representativeness of randomised groups}

All baseline descriptive variables of participants will be summarised by treatment arm. Continuous data will be expressed as $\mathrm{N} / \mathrm{mean} / \mathrm{SD} / \mathrm{min} / \mathrm{Q} 1$ (lower quartile)/median/Q3 (upper quartile)/max. Tabulations of frequencies for categorical data will include all possible categories and will display the number of observations in a category as well as the percentage (\%) relative to number of available values within the respective treatment group unless otherwise specified. The number of missing values is reported for both types of variables. This will allow a visual assessment of whether the randomisation procedure succeeded in producing comparable arms, and tests of statistical significance will not be undertaken between arms at baseline; rather the clinical importance of any imbalance will be noted.

The number of participants who receive the injection outside the planned visit window of 5 days or more will be reported by visit number and treatment arm. Also, the mean cumulative dosage by planned dose will be plotted by treatment arm and separately for those receiving monthly and fortnightly injections.

\section{Descriptive statistics for outcomes}

The distributions of all efficacy outcomes (in Table 1) will be presented in histograms (continuous/count) or bar charts (ordinal/binary) both overall and by group at each assessment point. A single table will be outputted with summary statistics for all outcomes by group and visit point. Furthermore, summary statistics will be plotted by line graphs for each outcome across time by intervention. Only participants with a completely recorded outcome will be used to calculate the summary measures.

\section{Analysis of primary efficacy outcome Primary analysis}

A linear mixed model will be used to obtain an estimate for the mean difference in objective SCORAD scores between the two treatment groups. Participant will be included as a random intercept (investigating adding a random slope on time), and time (investigating the possibility of linearising this effect across 8, 12, 16, 20 and 24 weeks), time-by-group interaction, baseline objective SCORAD score, $\operatorname{IgE}(\leq 1500,>1500)$ and age $(<10$ or $\geq 10$ years) as fixed effect. An overall treatment effect for objective SCORAD score at 24 weeks will be estimated.

The response $y_{i j}$ is the objective SCORAD score measurement for patient $i$ at time $t_{j}$. Both random intercept model (a) and random intercept and slope model (b) will be fitted as specified below: (a) $Y_{i j}=\beta_{0}+\beta_{1} T R T_{i}+\beta_{2} S C O R A D_{i}^{0}+\beta_{3} I g E_{i}+\beta_{4} A g e_{i}$

$$
+\beta_{5} t_{12}+\beta_{6} t_{16}+\beta_{7} t_{20}+\beta_{8} t_{24}+\beta_{9} t_{12}
$$$$
* T R T_{i}+\beta_{10} t_{16} * T R T_{i}+\beta_{11} t_{20} * T R T_{i}
$$$$
+\beta_{12} t_{24} * T R T_{i}+b_{i}+e_{i j}
$$

(b) $Y_{i j}=\beta_{0}+\beta_{1} T R T_{i}+\beta_{2} S C O R A D_{i}^{0}+\beta_{3} I g E_{i}+\beta_{4} A g e_{i}$

$$
+\beta_{5} t_{12}+\beta_{6} t_{16}+\beta_{7} t_{20}+\beta_{8} t_{24}+\beta_{9} t_{12}
$$$$
* T R T_{i}+\beta_{10} t_{16} * T R T_{i}+\beta_{11} t_{20} * T R T_{i}
$$$$
+\beta_{12} t_{24} * T R T_{i}+b_{1 i}+b_{2 i} t_{j}+e_{i j},
$$

where $j=$ time points $(8,12,16,20$ and 24 weeks), $i=$ participants,

$T R T_{i}$ : dummy variable $\left(T R T_{i}=0\right.$ or 1$)$ of patient $i$,

$\operatorname{Ig} E_{i}$ : dummy variable for $\operatorname{IgE}(=0$ or 1$)$ of patient $i$,

$S C O R A D_{i}^{0}$ : baseline SCORAD score of patient $i$,

Age $_{i}$ : dummy variable for age $(<10$ or $\geq 10$ years $)$ of patient $i$,

$t_{x x}$ : dummy variable for time $(=0$ or 1$)$ at time point $\mathrm{xx}$ weeks.

Where $b_{i}$ and $b_{1 i}$ are random intercepts, $b_{2 i}$ is random slopes, both $e_{i j}$ and $b_{1 i}$, following normal distributions. An unstructured covariance matrix will be used. Models will be fitted using residual maximum likelihood (REML). The estimated treatment effect at 24 weeks, $\beta_{1}$ $+\beta_{12}$, will be reported with $95 \%$ confidence intervals and corresponding $p$ value.

Model (a) will be the primary analysis model unless there is strong evidence for mis-specification of the model. The random slope model is less restrictive and possibly more realistic in its assumptions, i.e. the objective SCORAD score trajectories for each individual starting from a different level and following a different trend with a different slope. The primary interest is in determining whether $\beta_{1}+\beta_{12}$ is significant and whether this varies between the two models ( $\mathrm{a}$ and $\mathrm{b}$ ).

The conclusion of the trial will be based on this analyses.

\section{Planned sensitivity analyses}

To investigate the robustness of the results of the primary analysis we will undertake a number of sensitivity analysis:

1. Subsequent adjustment for cumulative use of potent topical steroids (continuous variable) at 24 weeks, alternative systemic therapy (yes/no) at 24 weeks, rescue medication (yes/no) at 24 weeks based on the primary model

2. An analysis of Complier Average Causal Effect (CACE) by a two-stage least squares instrumental variable regression would be performed for the 
primary endpoint as analysis based upon ITT may underestimated the effect of actually receiving the treatment [17]. Here, we defined 'compliers' as those who complete more than $50 \%$ of injections (that is injections received relative to injections planned for the 24-week study period in groups). Randomisation will be used as an instrumental variable for treatment received with the same covariates in primary analysis models.

\section{Analysis of secondary efficacy outcomes}

All analyses for secondary efficacy outcomes will be based on the ITT population and defined at week 24 unless specified otherwise. The missing data will be tackled according to the strategies mentioned above.

For each secondary outcome, we will adjust for the minimisation variables $\operatorname{IgE}(\leq 1500,>1500)$, age $(<10$ or $\geq 10$ years) and baseline data (as appropriate).

Treatment failure (binary) and alternative systemic therapy (binary) will be analysed using a logistic regression model. The estimated treatment effect (odds ratio) will be reported with $95 \%$ confidence intervals and corresponding $p$ value. Subjective SCORAD, EASI, PatientOriented Eczema Measure (POEM), Paediatric Allergic Disease Quality of Life Questionnaire (PADQLQ), (Children's) Dermatology Life Quality Index ((C)DLQI) scores and allergen IgE levels will be analysis using analysis of covariance (ANCOVA). The estimated treatment effect (mean difference) will be reported with 95\% confidence intervals and corresponding $p$ value. The number of skin-prick test reactivities, infective episodes of eczema count, and number of eczema exacerbations will be analysed by Poisson regression, Negative binomial regression or zero-inflated Poisson regression models after checking the distribution of the dependent variable by Pearson chi-square goodness-of-fit tests will ensure the selection of the correct statistical model. The estimated treatment effect (odds ratio) will be reported with 95\% confidence intervals and corresponding $p$ value. A summary of models for each of the outcomes can be found in Table 1.

\section{Analysis of safety outcomes}

Information on adverse events (AE) will be collected by means of spontaneous reports from patients and carers, clinical observation and clinical examinations and blood tests. Adverse events will be coded using terms chosen by the clinical investigators with reference to the Medical Dictionary for Regulatory Activities (MedDRA) at the Preferred Term level. Abnormal ranges for blood tests will be defined using the ranges specified by the laboratory processing the sample.

Adverse events will be tabulated overall by severity and type (AE, adverse reaction, unexpected adverse reaction, serious $\mathrm{AE}$, serious adverse reaction or unexpected serious adverse reactions). These will be summarised over the 48-week follow-up period and, where appropriate, by time of occurrence.. The numerator will indicate the number of affected participants at each time point from the SS population. The denominators will show how many participants were in the trial at the corresponding time point. If appropriate, the difference in proportion (95\% confidence interval) will be estimated and time-to-event curves by treatment arm will be plotted. All AE will be listed individually.

\section{Subgroup analyses}

A subgroup analysis is planned to investigate whether intervention effects differ between adherence, defined as the injections received relative to the injections planned for the 24 -week study period in groups $(\leq 50 \%,>50 \%$; $\leq 75 \%$, $>75 \%$; $\leq 90 \%,>90 \%)$. All subgroup analyses will be analysed using the same method as for the primary outcome. The results will be displayed by means of a forest plot.

\section{Software}

Data management: an online data collection system for clinical trials (MACRO; InferMed Ltd.) will be used. This is hosted on a dedicated server at Kings' Clinical Trial Unit. The CTU data manager will extract data periodically as needed and provide these in comma separated (.csv) format.

Statistical analysis: analysis will be performed using statistical software Stata, R or SAS.

\section{Tables and figures}

The SAP describes the conventions to be used for presenting results in text and in tables and figures. Those conventions are based on the International Conference on Harmonisation ( $\mathrm{ICH}$ ) guideline for reporting clinical trial results. The planned tables are:

- Analysis population by study centre and treatment group

- Withdrawals, protocol deviations and violations by treatment group

- Baseline demographic and clinical characteristics by treatment group

- Baseline blood/urine investigations by treatment group

- Descriptive analysis for primary efficacy outcomes by treatment group across study visits

- Inferential analysis for primary efficacy outcomes by treatment group

- Sensitivity analysis for primary efficacy outcomes by treatment group

- Secondary efficacy outcomes by treatment group

- $\mathrm{AE}$ by treatment group across study visits 
The planned figures are:

- Flow chart of participants through the study

- Bar chart/histogram for efficacy outcome over time by overall and treatment group

- Linear graph for efficacy outcome over time by treatment group

- Forest plot of effect of treatment on primary for all participants and for prespecified subgroups

This article presents the SAP for the ADAPT and should be read in conjunction with the trial protocol [5].

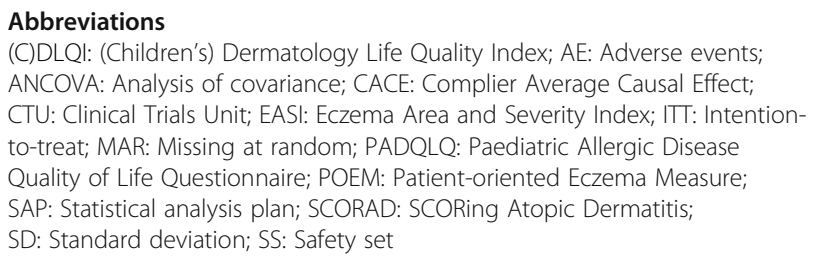

\section{Acknowledgements}

The authors wish to acknowledge the NIHR EME programme for funding the study (NIHR EME 11/14/24 ADAPT), Novartis for supplying the study drug and King's Clinical Trials Unit for providing support in the design and conduct of the trial (Caroline Murphy, Joanna Kelly) and the trial manager (Rahi Jahan). The Data Monitoring Committee (Professor Graham Roberts, Dr. Ahmed Massoud, Ms. Helen Moyses) and Trial Steering Committee (Dr. Nerys Roberts (chair), Dr. Mike Coren, Dr. Helen Cox, Professor John Harper, Mrs. Kathryn Humphreys, Dr. Susan Chan, Dr. Emma Wedgeworth, Professor Gideon Lack, Dr. Victoria Cornelius) for their advice and contributions to the protocol and statistical analysis plan. We wish to thank Dr. Kun Liu for his important contribution to the development of the statistical methods.

The study was designed and delivered in collaboration with the UK Clinical Research Collaboration (UKCRC)-registered King's College London Clinical Trials Unit. The study is supported by the NIHR Biomedical Research Centre which is based at Guy's and St. Thomas' NHS Foundation Trust and King's College London, and by a National Institute for Health Research comprehensive Biomedical Research Centre award to Guy's and St. Thomas' NHS Foundation Trust and King's College London.

\section{Funding}

This investigator-led trial is funded by the National Institute for Health Research Efficacy and Efficacy and Mechanism Evaluation Programme (NIHR EME 11/14/24) and the Guy's and St. Thomas' Charity. The study drug and placebo were supplied by Novartis. Novartis had no role in the design of the study or writing of the protocol or statistical analysis plan.

\section{Availability of data and materials}

The protocol and statistical analysis plan can be obtained by contacting the corresponding author. The study team will retain the exclusive use of data until publication of major outputs has been completed. Funding will be sought to make the data publically available.

\section{Authors' contributions}

TC participated in development of the trial protocol and statistical analysis plan and was responsible for preparing the manuscript. VC obtained grant funding, participated in development of the study design, trial protocol, statistical analysis plan and manuscript. SChan is the chief investigator, obtained grant funding, contributed to the study design and protocol and reviewed the manuscript. GL conceived the study, obtained grant funding, contributed to the study design and protocol and reviewed the manuscript. SCro participated in development of the trial statistical analysis plan, and contributed to, and reviewed, the manuscript. All authors read and approved the final manuscript.

\section{Authors' information}

Not applicable

\section{Competing interests}

The authors declare that they have no competing interests.

\section{Consent for publication}

Not applicable

\section{Ethics approval and consent to participate}

Ethical approval has been agreed and is being overseen by London Westminster NHS Research Ethics Committee (REC reference number: 11/ LO/0123). We will obtain informed consent from all participants in the study.

\section{Publisher's Note}

Springer Nature remains neutral with regard to jurisdictional claims in published maps and institutional affiliations.

\section{Author details}

${ }^{1}$ Department of Clinical Sciences, Liverpool School of Tropical Medicine, Liverpool L3 5QA, UK. ' Guy's and St. Thomas' NHS Foundation Trust, Westminster Bridge Road, London SE1 7EH, UK. ${ }^{3}$ King's College London, King's Health Partners, Asthma-UK Centre in Allergic Mechanisms of Asthma, Allergy and Respiratory Science, Guy's Hospital, London SE1 9RT, UK. ${ }^{4}$ Imperial Clinical Trials Unit, School of Public Health, Imperial College London, Stadium House, 68 Wood Lane, London W12 7RH, UK.

Received: 28 September 2016 Accepted: 4 May 2017

Published online: 23 May 2017

\section{References}

1. Lane JE, Cheyney JM, Lane TN, Kent DE, Cohen DJ. Treatment of recalcitrant atopic dermatitis with omalizumab. J Am Acad Dermatol. 2006;54(1):68-72.

2. Belloni B, Ziai M, Lim A, Lemercier B, Sbornik M, Weidinger S, Andres C, Schnopp C, Ring J, Hein R, Ollert M, Mempel M. Low-dose anti-lgE therapy in patients with atopic eczema with high serum IgE levels. J Allergy Clin Immunol. 2007;120:1223-5

3. Vigo PG, Girgis KR, Pfuetze BL, Critchlow ME, Fisher J, Hussain I. Efficacy of anti-lgE therapy in patients with atopic dermatitis. J Am Acad Dermatol. 2006;55:168-70.

4. Sheinkopf LE, Rafi AW, Do LT, Katz RM, Klaustermeyer WB. Efficacy of omalizumab in the treatment of atopic dermatitis: a pilot study. Allergy Asthma Proc. 2008:29:530-7.

5. Chan S, Cornelius VR, Chen T, Raulovic S, Wan M, Jahan R, Lack G. Atopic Dermatitis Anti-lgE Paediatric Trial (ADAPT): the role of anti-lgE in severe paediatric eczema: study protocol for a randomized controlled trial. Trials. 2017;16:136

6. Hindley D, Galloway G, Murray J, Gardener L. A randomised study of 'wet wraps' versus conventional treatment for atopic eczema. Arch Dis Child. 2006:91:164-8.

7. Howard $\mathrm{R}$, et al. Determining the minimum clinically important differences for outcomes in the DOMINO trial. Int J Geriatr Psychiatry. 2011;26(8):812-7.

8. Schram ME, Spuls PI, Leeflang MM, Lindeboom R, Bos JD, Schmitt J. EASI, (objective) SCORAD and POEM for atopic eczema: responsiveness and minimal clinically important difference. Allergy. 2012;67(1):99-106.

9. White IR, Horton NJ, Carpenter J, Pocock SJ. Strategy for intention to treat analysis in randomised trials with missing outcome data. BMJ. 2011;342:d40.

10. Kahan BC, Morris TP. Reporting and analysis of trials using stratified randomisation in leading medical journals: review and reanalysis. The BMJ. 2012;345:e5840.

11. Turner EL, Perel P, Clayton T, Edwards P, Hernandez AV, Roberts I, Shakur H, Steyerberg EW, CRASH trial collaborators. Covariate adjustment increased power in randomized controlled trials: an example in traumatic brain injury. J Clin Epidemiol. 2012;65:474-81.

12. Carpenter J, Kenward M. Missing data in randomised controlled trials-a practical guide. Birmingham: National Institute for Health Research. 2008; vol. Publication RM03/JH17/MK.

13. White $I R$, Royston $P$, Wood AM. Multiple imputation using chained equations: issues and guidance for practice. Stat Med. 2011;30:377-99. 
14. Hardt J, Herke M, Leonhart R. Auxiliary variables in multiple imputation in regression with missing $\mathrm{X}$ : a warning against including too many in small sample research. BMC Med Res Methodol. 2012;12:184.

15. White IR, Thompson SG. Adjusting for partially missing baseline measurements in randomized trials. Stat Med. 2005;24:993-1007.

16. Fayers PM, Curran D, Machin D. Incomplete quality of life data in randomized trials: missing items. Stat Med. 1998;17:679-96.

17. Sussman JB, Hayward RA. An IV for the RCT: using instrumental variables to adjust for treatment contamination in randomised controlled trials. The BMJ. 2010;340:C2073.

Submit your next manuscript to BioMed Central and we will help you at every step:

- We accept pre-submission inquiries

- Our selector tool helps you to find the most relevant journal

- We provide round the clock customer support

- Convenient online submission

- Thorough peer review

- Inclusion in PubMed and all major indexing services

- Maximum visibility for your research

Submit your manuscript at www.biomedcentral.com/submit 